\title{
Tecnologia na Atenção Primária: uma estratégia de apoio a gestão
}

\section{Technology in Primary Attention: a strategy to support management}

\author{
${ }^{1}$ Lucrecia Helena Loureiro lucreciahelena@gmail.com \\ ${ }^{1}$ Aline Silveira-Alves \\ ${ }^{2}$ Saulo Nogueira Hermosilla de Almeida \\ ${ }^{1}$ Ilda Cecília Moreira da Silva \\ ${ }^{3}$ Teresa Tonini
}

\section{RESUMO}

A atenção primária a saúde (APS), é o nível de atenção onde as pessoas são atendidas como sujeitos sociais. Atualmente é considerada a porta de entrada para o Sistema de Saúde Brasileiro. A partir da implantação do modelo assistencial voltado as famílias e a comunidade, em todo o território nacional foram surgindo novas unidades, cada vez mais próximas a comunidade, com uma nova proposta de reorientação da assistência buscando a consolidação dos princípios do Sistema Único de Saúde (SUS). Mas toda essa mudança de atendimento direcionada as famílias, acabou deixando uma lacuna nas formas de agendamento das consultas. Assim, este trabalho descreve um software para o agendamento, compatível com os princípios do SUS, com os critérios e parâmetros para o planejamento e programação de ações e serviços de saúde no âmbito do SUS. Propõe a integração entre o atendimento dos médicos e enfermeiras, com a automatização desses parâmetros, descrevendo a experiência do ensino-aprendizado em gerência de enfermagem com a construção de uma ferramenta informatizada para o agendamento das consultas. Constatou-se que o software é adequado, eficiente e que permite a interação dos dados, análise epidemiológica do território, agilidade no serviço e disponibilização de informações instantâneas sobre a capacidade operacional das unidades. O sistema desenvolvido apresentou-se como estimulo para melhor gestão e procurou auxiliar o processo de trabalho do enfermeiro com a incorporação de indicadores de saúde na prática cotidiana do trabalho do gerente.

Palavras-Chave: Software. Tecnologia em saúde. Estratégia Saúde da Família. Atenção Primária.

\begin{abstract}
Primary health care (PHC) is the level of care where people are served as social subjects. It is currently considered the gateway to the Brazilian Health System. From the implantation of the assistance model aimed at families and the community, new units emerged throughout the national territory, increasingly closer to the community, with a new proposal to reorient the assistance seeking to consolidate the principles of the Unified Health System ( SUS). But all this change of care directed to families, ended up leaving a gap in the ways of scheduling the consultations. Thus, this work describes software for scheduling, compatible with SUS principles, with the criteria and parameters for the planning and programming of actions and health services within SUS. It proposes the integration between the attendance of doctors and nurses, with the automation of these parameters, describing the teaching-learning experience in nursing management with the construction of a computerized tool for scheduling the consultations. It was verified that the software is adequate, efficient and allows data interaction, epidemiological analysis of the territory, agility in the service and availability of instantaneous information about the operational capacity of the units. The developed system was presented as a stimulus for better management and sought to assist the nurses'work process with the incorporation of health indicators into the daily practice of the manager's work.
\end{abstract}

Keywords: Software; Health technology; Family Health Strategy; Primary attention.

1 Centro Universitário de Volta Redonda (UniFOA)

2 Secretaria de Saúde de Volta Redonda

3 Professora Associada do Departamento de Enfermagem Fundamental; Coordenadora do Programa de Pós-Graduação em Enfermagem e Biociências (PPGENFBIO) 


\section{INTRODUÇÃO}

A implantação do Sistema Único de Saúde(SUS), em 1990, trouxe importantes mudanças no modelo de atenção à saúde pública no país; as unidades de saúde foram gradualmente disseminando-se por todo o território nacional e as equipes de saúde aproximando-se cada vez mais da população. Essa aproximação crescente deu estabilidade as propostas para reorientação do modelo assistencial vigente a época.

A base da reorientação do modelo assistencial foi a ênfase na atenção primária a saúde (APS), porta de entrada dos sistemas de saúde organizados. Percebe-se que essa ousada mudança centrada na comunidade e orientada as pessoas se consolidou em nosso país, e começou a tomar espaço com conhecimentos e práticas próprias. O arcabouço de formação do enfermeiro esse profissional tem contato com um estrato da realidade da população, aprendendo a habilidade de acolhimento, sem ter contato com a informatização e a evolução tecnológica na atenção primária a saúde.

Nossa sociedade tem sofrido impactos da evolução tecnológica digital em constante crescimento e avanço, o que nos remete a considerar os avanços, novas descobertas, invenções e inovações. Nesse contexto, as mudanças ocorrem muito rapidamente, novos conceitos surgem a cada segundo um novo software, um novo hardware e instantâneas e constantes transformações na área da informática. Por outro lado, as novas perspectivas do mercado de trabalho em todo o mundo tornaram imprescindível que as áreas de estudo e formação acompanhassem essa modernização e essa evolução tecnológica(LOPES et al.2011).

Num mundo em constante evolução tecnológica e modernização das atividades, tornou-se importante que as entidades de ensino, pesquisa e serviço de qualquer área de saber, sejam públicas ou privadas, se adaptassem às novas perspectivas do mercado de trabalho (BENITO et al.2009).

Porém, vale destacar que as tecnologias inovadoras, principalmente aquelas atreladas à informática ainda são vistas por muitos profissionais como um desafio e uma área ainda desconhecida, apesar de representar crescimento e perspectivas de atuação profissional.

Atualmente, o mundo vem enfrentando a transição de uma economia industrial para uma economia de informação, a qual transforma a natureza da riqueza. Nesse entendimento, o capital intelectual está se tornando relativamente mais importante do que o capital físico, a nova riqueza é a informação e o conhecimento é aplicado ao trabalho para criar valor, sendo que o sucesso é determinado pelo saber e não pelos bens materiais que se possui (PERES et al.2004).

Com o advento do computador surgiram os softwares, isto é, programas que permitem o uso e a aplicação de tecnologias da informática (VIGOSTSKY 2002). Muitos desses programas têm sido aplicados nos diferentes cenários de formação e de trabalho de profissionais da saúde, com destaque neste estudo para a enfermagem.

Utilizar os recursos do computador para promover educação é um desafio para enfermagem no Brasil, já que essa tecnologia continua sendo pouco explorada pelos profissionais. Ainda assim, destacamos que a Informática em Enfermagem é uma área de conhecimento com mais de 30 anos de aplicação e desenvolvimento (MARIN 2006).

Os computadores foram inicialmente utilizados pela enfermagem na década de 50, principalmente na área de maior atuação do enfermeiro à época, ou seja, nos hospitais. No início, o interesse pela capacidade do hardware e do software era o maior desafio. Estes profissionais até dominavam a forma de desenvolvimento das aplicações e as áreas gerenciais eram as mais favorecidas (MARIN 2006).

Nos dias atuais, contudo, ainda observamos que a enfermagem apresenta uma situação desfavorável em relação ao uso de sistemas de informação, automação e equipamentos tecnológicos (SANTOS 2010). 
É nesse contexto de confronto da era moderna, com a necessidade de ampliação das ferramentas (especialmente no ensino de gerência) para a melhoria no ensino na atenção primaria a saúde, que foi realizada esta pesquisa.

O presente estudo tem como objetivo relatar a experiência na construção desse Software para o gerenciamento do serviço de uma Unidade Básica Estratégia Saúde da Família.

\section{MÉTODOS}

Com base no contexto descrito e nos pontos relacionados a qualidade da forma de ensinar desenvolvemos uma estratégia de ensino- aprendizado em gerência de enfermagem na atenção primária a saúde, compatível com as ferramentas informatizadas já disponíveis na APS.

O presente estudo é um relato de experiência para o registro das situações relevantes que ocorreram durante a criação e a implementação da estratégia de ensino tendo como premissa a abordagem cognitivista postulada por Vygotsky. Entendemos que a relevância deste relato de experiência está na pertinência dos problemas apontados e no o nível aplicação dos procedimentos e resultados da intervenção em situações similares possíveis.

\section{RELATO DA EXPERIÊNCIA}

Tal protótipo funcional, objeto de análise neste trabalho, foi aplicado no ambiente de uma unidade de Estratégia Saúde da Família, em uma cidade do interior do estado do Rio de Janeiro.

Em meados do ano de 2014, desenvolvendo atividades gerenciais, especificamente na atenção primária a saúde, e observando a problemática desses unidades para definir as agendas dos profissionais que atendem na Estratégia Saúde da Família, por meio da experiência de 25 anos na enfermagem, decidiu-se pela construção de uma ferramenta que pudesse apoiar a gerência no processo diário do trabalho.

Ao final de uma ano de análise e estudo das formas de elaborar as agendas de atendimento na ESF, Optou-se pela preparação de um protótipo para redefinir o pano de fundo que compusesse a ferramenta gerencial. Entretanto, foi necessária a construção de planilhas como início do projeto e estas, por sua vez, deveriam ser avaliadas por um número de participantes para sua adequação.

Anteriormente à avaliação das planilhas e seleção dos participantes foi solicitada autorização formal da Instituição para pesquisa, e o projeto foi submetido à apreciação e aprovado pelo Comitê de Ética em Pesquisa da Universidade Federal do Estado do Rio de Janeiro, em Abril de 2016 e aprovada sob CAAE: 53528616.0.0000.5285.

Inicialmente, utilizou-se a última planilha do censo IBGE 12010, que apresenta os dados da população residente por bairro, sexo e faixa etária da população. Foi utilizada também uma matriz de dados para elaboração do aplicativo.

Ao final de um ano de aplicação das planilhas por um grupo de discentes em uma unidade ambulatorial, constituída por 03 equipes da ESF, com atendimento de pessoas de ambos sexos e todas as faixas etárias e perfil heterogêneo de saúde, foi necessário uma apreciação detalhada dos achados.

Porém, ao analisarmos o preenchimento das planilhas surgiu a necessidade de uma abordagem maior e uma adequação das informações colhidas, para uma melhor compreensão dos dados pela gerência da unidade. 
Verificou-se em loco que as agendas dos profissionais em algumas unidades estavam em propriedade da recepção, outras com os próprios profissionais, e a gerência considerada fundamental nesse processo era o ator menos envolvido nessa questão. Baseados nessas premissas, decidimos elaborar mais três novas planilhas para atender a gerência na análise da capacidade operacional da unidade a luz dos dados epidemiológicos.

Como critérios, desenvolveu-se a segunda planilha para distribuição etária da população total da área de abrangência, ou seja, o $N^{\circ}$ de habitantes estimados no ano. Na terceira organizou-se a distribuição da população segundo categoria programática, na quarta e última planilha elaborada, tratou-se da Análise da Capacidade Operacional da Unidade.

A elaboração da agenda, com embasamento científico e não puramente uma alocação de profissionais durante a semana, com agendas superlotadas e muitas vezes programadas pela recepcionista deixariam de existir (LOUREIRO et al. 2010).

Para auxiliar na verificação, validação e teste da efetividade das planilhas, que seriam os protótipos do Software, optou-se por capacitar 30 discentes do $8^{\circ}$ período do Curso de Graduação em Enfermagem, em estagio supervisionado na disciplina de Gerencia da Atenção Primária a Saúde. Com o objetivo de melhorar a compreensão e facilitar o preenchimento das planilhas, foi idealizado um Kit, com as 4 planilhas e entregues aos discentes selecionados.Optou-se por ministrar 4 aulas perfazendo um total de 16 horas, para finalização da tarefa pelos discentes, onde todos foram previamente orientados quanto a forma de preenchimento e análise dos dados. Com base no perfil epidemiológico da população estudada, foi possível a construção das agendas programáticas da equipe de saúde da ESF.

Concluída essa etapa, os discentes responderam a um questionário, para avaliar e identificar as dificuldades encontradas no decorrer da tarefa. Na avaliação das planilhas uma das perguntas do questionário foi sobre as dificuldades encontradas no preenchimento, 62\% responderam que a dificuldade estava na falta de prática e de conhecimento especifico; 23\% responderam que após a explicação do objetivo da planilha, não houve dificuldade no preenchimento e que elas são de fácil entendimento; 15\% responderam a pergunta com resposta incoerente, concluímos que os alunos não entenderam a pergunta.

A análise desses dados permite afirmar que o modelo de planilha elaborado auxilia no aprendizado de gerência e organiza o processo de trabalho gerencial da ESF, por auxiliar na interpretação das informações e, consequentemente, facilita a elaboração das agendas dos profissionais envolvidos no processo. Entretanto, observou-se dificuldades dos estudantes para a elaboração das agendas, fato esse evidente na prática; somente 60\% dos alunos conseguiram organizar o agendamento de forma clara e objetiva.

\section{A CONSTRUÇÃO DO SOFTWARE}

A construção do software obedeceu as seguintes fases: 1) elaboração do objetivo; 2) decisão do público-alvo; 3) seleção do referencial pedagógico; 4) eleição do referencial teórico; 5) escolha do conteúdo; 6) desenvolvimento do software e 7) avaliação do software, explicitadas a seguir.

\section{1 Finalidade da Utilização do Software}

Capacitar o profissional para uma nova concepção de gerência com o planejamento correto das agendas estabelecendo a capacidade operacional da unidade à luz de dados epidemiológicos. 


\section{2 Opção pelo público-alvo}

O público-alvo refere-se a todos os profissionais na função gerencial, em especial da Enfermagem. O seu uso poderá ser extensivo aos profissionais da área da saúde pública em função de gerencia e gestão.

\section{3 Referencial Pedagógico}

Utilizou-se como premissa para elaboração do software, a abordagem cognitivista postulada por Vygotsky. Nesta abordagem, o ato ou o processo de conhecer, a percepção visual e audível, a aprendizagem, é entendida como pratica, e não como, representação, portanto a aprendizagem na abordagem cognitivista considera as formas pelas quais os indivíduos lidam com os estímulos ambientais, organizam dados, percebem e resolvem problemas, adquirem conceitos e empregam símbolos verbais, portanto e considerada predominantemente interacionista (VIGOSTSKY 2002).

Baseando neste referencial, o desenvolvimento do software, permitiu a construção de recursos individualizados permitindo o comando do próprio profissional, revisando todos os conhecimentos adquiridos na academia, com mediação do processo de aprender a aprender, estimulação ao autodesenvolvimento e ao controle próprio da aprendizagem, exercitação da cultura lúdica e estimulação sensorial(VIGOSTSKY 2002).

\section{4 Referencial Teórico}

Atualmente as tecnologias estão presentes na sociedade da informação em que vivemos e são indispensáveis para a comunicação, a formação, a prática profissional, em suma, para a vida. No contexto da formação em saúde, o uso de tecnologias é uma alternativa na busca de melhorar o processo de ensino-aprendizagem e preparar os estudantes para viverem nesta sociedade em constante evolução (LOUREIRO et al. 2010).

Poderíamos definir tecnologias de diversas maneiras, no entanto podemos dizer de maneira simples que tecnologias podem ser objetos, instrumentos, aparelhos eletrônicos, enfim, todos os recursos que venham facilitar nossas vidas e em alguns momentos se tornam indispensáveis (HENZ 2008).

Considerando que todas as organizações utilizam alguma forma de tecnologia para executar suas operações e/ou realizar suas tarefas, observa-se que os avanços tecnológicos criaram mudanças em várias áreas da vida moderna. Na área de assistência à saúde, especificamente, na área de enfermagem, novos e complexos desafios são enfrentados relativos à implementação, utilização, avaliação e desenvolvimento destas novas tecnologias (LOPES et al. 2011).

\section{5 Escolha do conteúdo}

A partir do estudo do referencial pedagógico e teórico definiu-se a utilização e a aplicação de novas tecnologias da informática, com programas que permitem o uso e aplicação gerencial, que são desenvolvidos em função de clientes específicos. Os computadores contribuem para a melhoria da educação, da qualidade de docentes e administradores nas instituições provedoras de serviços de saúde(LOPES et al. 2011).

O acompanhamento do progresso das tecnologias da informática e nossas experiências na gerencia em saúde coletiva reforçaram a opinião de associar as possibilidades da informática no cotidiano do gerente das Unidades da Estratégia de Saúde da Família. 


\section{6 Desenvolvimento do Software}

O desenvolvimento de um projeto multimídia envolve um grande número de variáveis e apesar dos avanços tecnológicos permitirem a criação de multimídias de qualidade, muito esforço, dedicação e tempo são requeridos de que se propõe a desenvolvê-los (BERNARDO 1996)

A interface e a estrutura que permitem o dialogo entre o software e o profissional, é estabelecido. Como o homem percebe o mundo por meio do sistema sensório, o planejamento de uma interface deve enfatizar os sentidos visuais e auditivos (VIGOTSKY 2002). Como essa tecnologia tem sido pouco explorada pela enfermagem, é necessário estar alerta às mudanças que já são presentes e para as que virão no futuro (MARQUES et al. 2010).

Na construção do software foi utilizado o programa Microsoft Access. Foi desenvolvido para funcionar em plataforma Microsoft Windows na versão “XP” 2003. O sistema dá possibilidade de utilização em rede e, o equipamento mínimo necessário é microcomputador da linha PC-AT 286 ou superior, com monitor (monocromático ou colorido), possuindo no mínimo $640 \mathrm{~Kb}$ de memória RAM, com Winchester de $30 \mathrm{Mb}$ ou mais de capacidade, dependendo do volume de dados de interesse da instituição usuária, sendo que 1,8 Mb serão ocupados pelo sistema.

O produto final deste estudo resultou em um software denominado "Gestão do Serviço de Enfermagem na Estratégia Saúde da Família”, que engloba á organização das informações e apoio ao planejamento gerencial das unidades de ESF. Possui oito telas, a saber: (1) Tela de acesso; (2) Tela inicial do software; (3) Tela de cadastro de bairro; (4) Planilha adaptada do censo IBGE-2000; (5) Relatório - População residente por bairro; (6) Distribuição etária da população total da área de abrangência; (7) Distribuição da população segundo a categoria programática e; (8) Análise da capacidade operacional da unidade.

\section{7 Avaliação do software}

Para avaliação do software foram convidados 33 enfermeiros que ocupavam cargo de gerentes na ESF. Todos os avaliadores atenderam ao critério de inclusão: um ano ou mais de experiência em gerência na ESF ou título de especialista nesta área.

Os participantes receberam um notebook com o software para que cada enfermeiro pudesse estudar o programa multimídia por até duas horas, por meio de navegação individual. Logo após essa etapa, solicitou-se aos gerentes que procedessem a uma avaliação do programa, respondendo um questionário com sete afirmativas, baseado na escala de Likert, e que respondessem sobre o software com as respectivas opções: ótimo, muito bom, bom, regular e ruim.

Sentiu-se a necessidade da avaliação do produto também por um especialista na área de informática, assim, foi feita a avaliação por um analista de sistemas, que conferiu outros aspectos pertinentes à parte de informática Ambos os instrumentos de avaliação foram fundamentados em outros estudos relacionados a softwares educacionais. Todos os avaliadores entregaram o instrumento preenchido e gastaram em média 1 hora e 42 minutos para avaliar o software.

Os itens avaliados no software receberam conceitos ótimo e muito bom de todos os avaliadores, sendo, portanto, validado seu conteúdo e sua aplicabilidade na ESF. Com relação às tarefas de implantação, revisão pós-implantação e distribuição da fase de implementação, não fizeram parte deste estudo e serão contempladas em estudos posteriores. 


\section{RESULTADOS E DISCUSSÕES}

O software teve por meta apresentar aos gerentes da ESF um recurso interativo a ser usado como ferramenta que auxiliassem o planejamento estratégico das unidades, à luz de dados epidemiológicos.

Vive-se momentos de crescente evolução e renovação tecnológica. Além disso, as tecnologias e os sistemas de informação tem-se aprimorado de forma abrangente a cada segundo.

Como é sabido, vive-se numa época de crescente evolução e renovação em tudo que diz respeito à tecnologia. Os idealizadores de software e hardware lançam seus produtos em tempo recorde no mercado.

Com o crescente aumento na utilização de software livre, principalmente em países em desenvolvimento, surge a necessidade de se migrar para soluções livres e multiplataformas (SANTOS 2010).

Entende-se por soluções livres, aquelas produções de software e artefatos de software sem custo de aquisição e suporte, usado de forma livre.

Atualmente, o Brasil destaca-se por adotar essas soluções gratuitas, principalmente no setor público. O crescente aumento por práticas de software livre é de notável relevância (SANTOS 2010).

A construção do software compreendeu os meses de maio a dezembro de 2016. O sistema contou com a participação efetiva das pesquisadoras e de uma técnica da área de informática. A foto da tela inicial do programa foi idealizada pelas pesquisadoras e as telas subsequentes foram construídas pela técnica em informática que participou do referido trabalho.

Durante cinco semanas aproximadamente, foram feitas reuniões para troca de informações e sugestões sobre as telas, os objetivos do trabalho, o conteúdo trabalhado, o perfil dos usuários e principalmente a tecnologia que seria empregada no desenvolvimento do software. Depois de finalizada essa etapa, elaborou-se um questionário que serviria como avaliação do produto.

O desenvolvimento do Software Gestão do Serviço de Enfermagem na Estratégia Saúde da Família, almejou construir uma interface simples, com bastante praticidade, fazendo com que as telas do sistema aproximasse da realidade do usuário final. Sua construção obedeceu as estapas de elaboração dos objetivos, escolha detalhada do referencial teórico, estabelecimento de meta e seleção do conteúdo e por fim desenvolvimento do sistema e sua aplicabilidade foram totalmente baseados nas práticas gerenciais desenvolvidas no âmbito da saúde coletiva, especificamente na ESF.

O enfermeiro gerente entrará com seu login e senha individual, antecipadamente cadastrado no sistema, com o objetivo de responsabilizar por seus registros.

Logo após a digitação do login e da senha, o sistema abre uma nova janela, nessa área do sistema, o profissional pode visualizar todas as telas existentes, elaborar as agendas dos profissionais, planejamento estratégico para a unidade, com a leitura do perfil epidemiológico da população e ainda acompanhar a evolução e crescimento populacional ao longo dos anos.

Nessa interface, o primeiro contato com o sistema, o gerente preencherá a planilha com os dados de sexo e faixa etária e obter as informações referentes ao perfil epidemiológico da população, com base nos conhecimentos de administração e gerenciamento poderá visualizar quais as intervenções de promoção à saúde poderá ser implementada para a população adstrita. 
Sabe-se que muito tem de ser feito para o acompanhamento dessas novas tecnologias e inovações, ressaltando a necessidade da efetiva avaliação dos mesmos antes de seu uso como instrumento de ensino. Pesquisas ainda precisam ser desenvolvidas sobre avaliação de softwares educacionais, área sabidamente recente e aberta ainda a inúmeros estudos (LOPES et al. 2011; Borges 1998; LOPES 2004.)

Com isso, espera-se que os profissionais que utilizarem o sistema para auxiliar no processo de gerenciar, além de facilitar a obtenção dos dados, tenham uma útil ferramenta de trabalho.

O software de forma geral foi muito bem aceito pelos avaliadores e acredita-se na relevância dos dados por ele produzidos.

\section{CONSIDERAÇÕES FINAIS}

A relevância dos sistemas de informação como apoio, na área administrativa de gerencia e a gestão do trabalho em saúde torna-se evidente. Todas as áreas do campo da saúde coletiva podem usufruir das ferramentas do software como auxilio no processo de trabalho, estas ferramentas são capazes de identificar os problemas no processo de trabalho das equipes das ESF, potencializando a resolutividade das situações e necessidades no processo de gerenciar.

Como primeira experiência, todas as dificuldades de principiantes na tarefa de construir um software, deu a perceber o grande desafio, entender a aplicabilidade do software no processo de gerenciar, a busca por teses e dissertações que abordassem informática e especificamente a construção e produção de software, como requisito para auxiliar na trajetória metodológica do presente trabalho.

O software foi elaborado para utilização como instrumento no processo de gerenciar das Unidades de Estratégia Saúde da Família. O mesmo foi analisado antes e após a sua construção, o que demonstrou significativa aplicabilidade, como se comprovou nos resultados obtidos na avaliação das planilhas e nas avaliações do software em questão; ressalta-se a necessidade de implantação para que o produto possa ser comprovadamente validado o produto. Os comentários positivos do software, de forma geral, contribuíram para a finalização desta etapa do estudo.

Assim, a avaliação de um produto torna-se um processo permanente e interminável. Após o termino de um software gerencial e sua disponibilização ao uso, o feedback dos profissionais pode fornecer ideias para melhorias a serem incorporadas em versões revisadas posteriormente.

É conveniente ressaltar que os sistemas de informação que disponibilizam dados de forma organizada e de fácil acesso, tronam-se recursos tecnológicos capazes de auxiliar efetivamente no processo de trabalho dos profissionais de saúde, auxiliando na tomada de decisões, consequentemente tem-se profissionais mais capacitados e capazes de mudar a realidade e melhorar o atendimento das ESF, através da inovação no processo de trabalho.

Com isso, espera-se que os profissionais que atuam como gerentes da ESF, tenham uma útil ferramenta de trabalho. 


\section{REFERÊNCIAS}

BENITO, G.A.V. \& LICHESKI, A.P. Sistemas de Informação apoiando a gestão do trabalho em saúde. Rev Bras Enferm, Brasília 2009 maio-jun; 62(3): 447-50.

BERNARDO, V. Desenvolvimento de programa multimídia aplicado ao ensino da medicina. Tese de Mestrado, EPM/UNIFESP, 1996.

BORGES P.R.T. Qualidade de “software” educacional: critérios para validação de treinamentos multimídia utilizados em educação à distância. Tecnol Educ. 1998;26(140):11-7 .

BOTTI N.C.L.; CARNEIRO, A.L.M.; ALMEIDA, C.S.; PEREIRALL, C.B.S. Construção de um software educativo sobre transtornos da personalidade. Rev Bras Enferm, Brasilia 2011 nov-dez; 64(6): 1161-6.

HENZ, C. C. O uso das tecnologias no ensino-aprendizagem da matemática. 2008. Monografia (Graduação em Matemática) - Departamento de Ciências Exatas e da Terra, Universidade Regional Integrada do Alto Uruguai e das Missões. Erechim.

LOPES, A.C.C.; FERREIRA, A.A.; FERNANDES, J.A.L.; MORITA, A.B.P.S; POVEDA, V.B.; SOUZA, A.J.S. Construção e avaliação de software educacional sobre cateterismo urinário de demora. Rev Esc Enferm USP. 2011; 45(1):215-22.

LOPES, M.V.O.; ARAUJO, T.L. Avaliação de alunos e professores acerca do software "Sinais Vitais". Rev Esc Enferm USP. 2004;38(4):438-47.

LOUREIRO, L H. Ensino de gerência em saúde coletiva e a educação permanente dos profissionais da ESF : $o$ uso do software educativo / Lucrecia Helena Loureiro. - Volta Redonda: UniFOA, 2010. 68 f. Dissertação (Mestrado Profissional) -- Centro Universitário de Volta Redonda -- UniFOA. Pós-graduação em Ensino em Ciências da Saúde e do Meio Ambiente, 2010. Orientadora: Professor Dra. Márcia Ribeiro Braz.

MARIN, H.F.; CUNHA, I.C.K.O. Perspectivas atuais da Informática em Enfermagem. Rev Bras Enferm 2006 maio-jun;59(3): 354-7.

MARQUES, I.R.; MARIN, H.F. Enfermagem na web: o processo de criação e validação de um web site sobre doença arterial coronariana. Rev Latino-am Enfermagem 2002 maio-junho; 10(3):298-307.

NUNES, M. O.; TRAD, L.B.; ALMEID. A.B.A.; HOMEM, C.A; MELO, C.I.C. O agente comunitário de saúde:construção da identidade desse personagem híbrido e polifônico. Cad. Saúde Pública, Rio de Janeiro, 18(6):1639-1646, nov-dez, 2002.

NUTO, S.A.S; NORO, L.R.A; CAVALSINA, P.G.; COSTA, I.C.C.; OLIVEIRA, A.G.R.C. O processo ensinoaprendizagem e suas consequências na relação professor-aluno-paciente. Ciência \& Saúde Coletiva. v. 11, n. 1, p. 89-96, 2006.

PERES H.H.C. \& KURCGANT P. O ser docente de enfermagem frente ao mundo da informática. Rev Latino-am Enfermagem 2004 janeiro-fevereiro; 12(1):101-8.

SANTOS, S.R. Informática em enfermagem: desenvolvimento de software livre com aplicação assistencial e gerencial. Rev Esc Enferm USP 2010; 44(2):295-301.www.ee.usp.br/reeusp/

SHIRATORI, K. SILVA, R.C.L., SILVA, C.R.L. Pesquisa em Saúde - termos e expressões. Rio de Janeiro: Águia Dourada.276p. 2009.

VIGOSTSKY L.S. Uma perspectiva histórico-cultural da educação. Petrópolis: Vozes; 2002. 